\title{
Transplantation of Neonatal Mouse Cardiac Macrophages into Adult Mice
}

\author{
Yandong $\mathrm{Li}^{1}$, Jie Feng ${ }^{1}, \mathrm{Yan} \mathrm{Li}^{1}$, Jianqiu Pei ${ }^{1}$, Shengshou $\mathrm{Hu}^{1}, \mathrm{Yu} \mathrm{Nie}{ }^{1,2}$ \\ ${ }^{1}$ Fuwai Hospital \& Chinese Academy of Medical Sciences, State Key Laboratory of Cardiovascular Disease, Cardiovascular Institute, National Center for \\ Cardiovascular Diseases, Peking Union Medical College ${ }^{2}$ National Health Commission Key Laboratory of Cardiovascular Regenerative Medicine, Fuwai \\ Central-China Hospital, Central-China Subcenter of National Center for Cardiovascular Diseases
}

\section{Corresponding Author}

Yu Nie

nieyu@fuwaihospital.org

\section{Citation}

Li, Y., Feng, J., Li, Y., Pei, J., Hu, S., $\mathrm{Nie}, \mathrm{Y}$. Transplantation of Neonatal Mouse Cardiac Macrophages into Adult Mice. J. Vis. Exp. (169), e62108, doi:10.3791/62108 (2021).

\section{Date Published}

March 20, 2021

DOI

$10.3791 / 62108$

\section{URL}

jove.com/video/62108

\section{Abstract}

In an injured neonatal myocardium, macrophages facilitate cardiomyocyte proliferation and angiogenesis and promote heart regeneration. The present study reveals that transplantation of neonatal cardiac macrophages recruited by injury promotes adult heart regeneration after myocardial infarction with improvement of cardiac function and cardiomyocyte proliferation. The results indicate that neonatal cardiac macrophage transplantation could be a promising strategy for cardiac injury treatment. Here, we provide the technical details, including the isolation of neonatal cardiac macrophages from apical resection-injured neonatal mouse hearts, the transplantation of macrophages into myocardial-infarcted adult mice, and the estimation of heart regeneration after a macrophage graft.

\section{Introduction}

Heart regeneration is a promising strategy to recover cardiac function after cardiac injury and to protect against heart failure ${ }^{1,2,3}$. Following myocardial injury, macrophages infiltrate the injured heart and have been explored as the key factors during neonatal heart regeneration ${ }^{4,5,6}$. Besides clearing necrotic cellular debris and inducing inflammation, macrophages promote angiogenesis ${ }^{5}$ and cardiomyocyte proliferation ${ }^{7}$ after neonatal mice myocardial infarction.

Our previous study illustrates that transplantation of neonatal cardiac macrophages isolated from injured neonatal heart enhances adult heart regeneration ${ }^{7}$, indicating that neonatal cardiac macrophage transplantation could be a promising strategy to treat cardiac injury. Here we provide the technical details, including isolation of neonatal cardiac macrophages from apical resection-injured neonatal mouse hearts, the 
transplantation of macrophages in to myocardial-infarcted adult mice, and the estimation of heart regeneration after macrophage graft (Figure 1).

\section{Protocol}

All experiments were conducted in accordance with the Guide for the Use and Care of Laboratory Animals. All animal protocols were approved by the Institutional Animal Care and Use Committee (IACUC), Fuwai Hospital, Chinese Academy of Medical Sciences.

\section{Apical resection operation in neonatal 1-day-old CX3cr1 ${ }^{\text {GFP/+ }}$ mouse ( $C 57 B L / 6$ background)}

\section{Anesthesia}

1. Take all the mouse pups out from the cages and put them in a clean dry box.

2. Embed each mouse in the ice for about 2-3 minutes. Cover the pup with gauze to avoid frostbite.

3. Identify sufficient anesthesia by observing for the following signs: pale skin and lack of limb movement.

2. Thoracotomy

1. Precool the bronze operating platform overnight at $-20^{\circ} \mathrm{C}$.

2. Take the anesthetized mouse out of the ice box and put it on a bronze operating platform. Anchor the mouse on the operating platform in a supine position using medical adhesive tape.

3. Put the operating platform with the mouse on it under a stereoscope.

4. Disinfect the mouse chest using a prep pad soaked with betadine and $70 \%$ alcohol.
5. Incise the skin with a $1 \mathrm{~cm}$ cut at the fourth intercostal area of the chest cavity and then separate the intercostal muscles using microsurgical scissors until the heart is accessed.

6. Alternately press the chest and abdomen with the help of two forceps until the heart is exteriorized out of chest without any mechanical damage.

7. Set the ribs below and above the small incision as a natural fixation to immobilize the heart.

3. Ventricular apex resection

1. Locate the apex of the left ventricle. Cut a $1 \mathrm{~mm}$ diameter of ventricular apex tissue using iridectomy scissors under a stereoscope.

2. Confirm that the left ventricular chamber is exposed, and begin oozing.

3. Gently press the heart back to chest cavity using a cotton swab.

4. Suture the muscles, ribs, and skin using 8-0 Prolene sutures.

5. Clean the mouse thoroughly after the operation.

4. Post-operation care

1. Transfer the mouse from the operating platform to a $37{ }^{\circ} \mathrm{C}$ heating blanket to warm up the body immediately after the operation.

2. Confirm anabiosis of the mouse by observing for the following signs: spontaneous respiration restoration, skin color change from pale to pink, and movement of limbs.

3. Take the operated mouse back to its mother once it has recovered. 
4. Mingle the operated mouse with its mother's nesting materials if necessary.

NOTE: It is important to remove all the 1-day-old pups from the mother at once and then return them all at once after all the pups are recovered.

\section{Preparation of the neonatal cardiac macrophage suspension}

NOTE: All these experimental procedures should be carried out in a dark place and under sterile conditions.

1. Harvest the heart of a neonatal $C \times 3 c r 1^{G F P /+}$ mouse 1day after apical resection

1. Euthanize the $C \times 3 c r 1^{G F P /+}$ mouse one day after the apical resection. Use an excess of carbon dioxide and decapitate the mouse sequentially to apply euthanasia.

2. Take the heart out of the chest and immerse it in a $10 \mathrm{~cm}$ dish with PBS.

3. Cut the vessels and remaining connective tissue away from the ventricles. Cut the auricular appendix and outflow tract away from the heart.

4. After the heart stops beating, cut the heart into 1-2 $\mathrm{mm}^{3}$ pieces in PBS buffer using microsurgical scissors (Figure 2A).

2. Neonatal mouse heart dissociation

1. Transfer the harvested heart tissue to a tube containing $2.5 \mathrm{~mL}$ of preheated enzyme mix (Table of Materials), and tightly close the tube.

2. Invert the tube and place it with the cap down (Figure 2B).
3. Run the neonatal heart dissociation program (Table of Materials).

4. Detach the tube from the dissociator after the program is completed.

5. Add $7.5 \mathrm{~mL}$ of $1 \times$ DMEM with $10 \%$ FBS to the tube.

6. Filter and transfer the suspension to a $15 \mathrm{~mL}$ centrifuge tube (Figure 2C). Centrifuge the cell suspension at $300 \times \mathrm{g}$ for 5 minutes.

7. Resuspend the cell pellet in $1 \mathrm{~mL}$ of blood cell lysis solution and incubate for 2 minutes at room temperature.

8. Add $5-10 \mathrm{~mL}$ of PBS buffer to the suspension and centrifuge at $300 \times \mathrm{g}$ for 5 minutes.

9. Resuspend the cell pellet in $1 \mathrm{~mL}$ of DMEM with $10 \%$ FBS.

3. Neonatal cardiac macrophage isolation

1. Sort GFP ${ }^{+}$neonatal cardiac macrophages by FACS. Receive the sorted macrophages in a sterile tube containing $500 \mu \mathrm{L}$ of DMEM with $10 \%$ FBS.

2. Count the $\mathrm{GFP}^{+}$macrophages (Figure 2D). Resuspend the macrophage in DMEM with $10 \%$ FBS at the concentration of $1 \times 10^{6}$ macrophages per $200 \mu \mathrm{L}$ of DMEM for later injection.

NOTE: The number of $\mathrm{GFP}^{+}$macrophages is small in one neonate (approximate $1 \times 10^{5}$ ); therefore, at least 10 neonates should be used to obtain enough macrophages for each adult mouse injection. 


\section{Neonatal cardiac macrophage transplantation}

1. Perform a myocardial infarction operation on an adult (6-8-week-old) male C57BL/6 mouse by ligation of the left anterior coronary artery ${ }^{8}$.

2. Put the operated mouse on a $37^{\circ} \mathrm{C}$ heated blanket until it recovers.

3. Intravenously inject $200 \mu \mathrm{L}$ of DMEM with $1 \times 10^{6} \mathrm{GFP}^{+}$ neonatal cardiac macrophages into the infarcted adult mouse after the operation (approximate 6 hours later) via the tail vein.

4. Send the mouse back to a clean cage.

\section{Result evaluation}

1. Injection efficiency validation

1. Anesthetize the operated mouse and harvest the heart 7 days after the myocardial infarction operation.

2. Immerse the heart in the pre-cooled PBS buffer.

3. Fix the heart tissue with $4 \%$ polyformaldehyde for 72 hours at room temperature with shaking.

4. Dehydrate the heart tissue in dimethylbenzene and ethanol.

5. Embed the heart tissue in paraffin and slice it into sections with a thickness of $5 \mu \mathrm{m}$.

6. Perform the standard immunofluorescence staining protocol $^{1}$. Use $\alpha$-actinin to mark cardiomyocytes.

7. Detect the $\mathrm{GFP}^{+}$macrophages in the heart receiving transplantation.

8. Perform the standard immunofluorescence staining protocol $^{1}$. Use $\alpha$-actinin and $\mathrm{pH} 3$ to mark cardiomyocytes and cell proliferation, respectively (Figure 3A).

2. Cardiac repair evaluation after the transplantation

1. Use echocardiography on the operated mouse one month after the operation ${ }^{1}$.

2. Analyze the cardiac function by comparing the left ventricular ejection fraction and fractional shortening in different groups (Figure 3B).

3. Anesthetize the operated mouse and harvest the heart.

4. Repeat steps 4.1.2-4.1.5.

5. Perform a Masson's staining protocol.

6. Analyze the infarcted area after macrophage injection (Figure 3C).

\section{Representative Results}

The protocol described here is summarized in a flow chart (Figure 1). We performed apical resection operation on 1day-old $C \times 3 c r 1^{G F P /+}$ mouse. As shown in Figure 2A, the neonatal $C \times 3 \mathrm{Cr}^{\mathrm{GFP} /+}$ mouse was anchored on the operating platform under the stereoscope after anesthesia. We applied pressure alternately on the mouse chest and abdomen with the help of two forceps, which can be set up as a tract to guide the heart popping out of the chest. Any extra mechanical damage to the heart that may affect heart regeneration should be avoided. The heart was immobilized by the surrounding chest tissues, which facilitated the operation on the myocardium. We found that cutting off less than a $1 \mathrm{~mm}$ diameter of the resected apex tissue by iridectomy scissors was appropriate when the left ventricular chamber began oozing. Successful induction of apical resection model is necessary for macrophage transplantation ${ }^{8,9}$. 
We sorted the $\mathrm{GFP}^{+}$macrophages strictly following a neonatal heart dissociation protocol ${ }^{1}$ (Figure 2).

Neonatal cardiac macrophages were injected into the myocardial infarcted adult mouse heart shortly after the isolation. In order to confirm the efficiency of the macrophage transplantation, we performed immunofluorescence staining at 7 days after the injection. The results showed that GFP ${ }^{+}$ macrophages could be found in the adult myocardial infarcted mouse heart, indicating the successful transplantation of neonatal cardiac macrophage. We employed coimmunostaining of $\mathrm{pH} 3$ with $\alpha$-actinin. The co-localization was considered to be cardiomyocyte proliferation. The results illustrated that the number of proliferative cardiomyocytes was upregulated in the macrophage-injected group, indicating that adult cardiomyocyte proliferation ability was enhanced after the transplantation (Figure 3).

Adult mouse heart regeneration was evaluated 1 month after the transplantation. We performed echocardiogram on the adult mouse and found that macrophage injection could enhance the cardiac function after myocardial infarction. Masson's staining was performed, and the results illustrated that infarcted area was remarkably reduced after the neonatal cardiac macrophage transplantation (Figure 3). All these results demonstrated that neonatal cardiac macrophage transplantation promotes adult mouse heart regeneration and cardiomyocyte proliferation. 

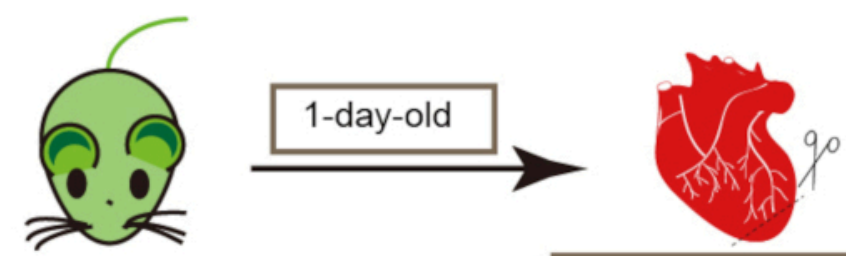

Cx3cr1GFP/+ mouse

Apical Resection
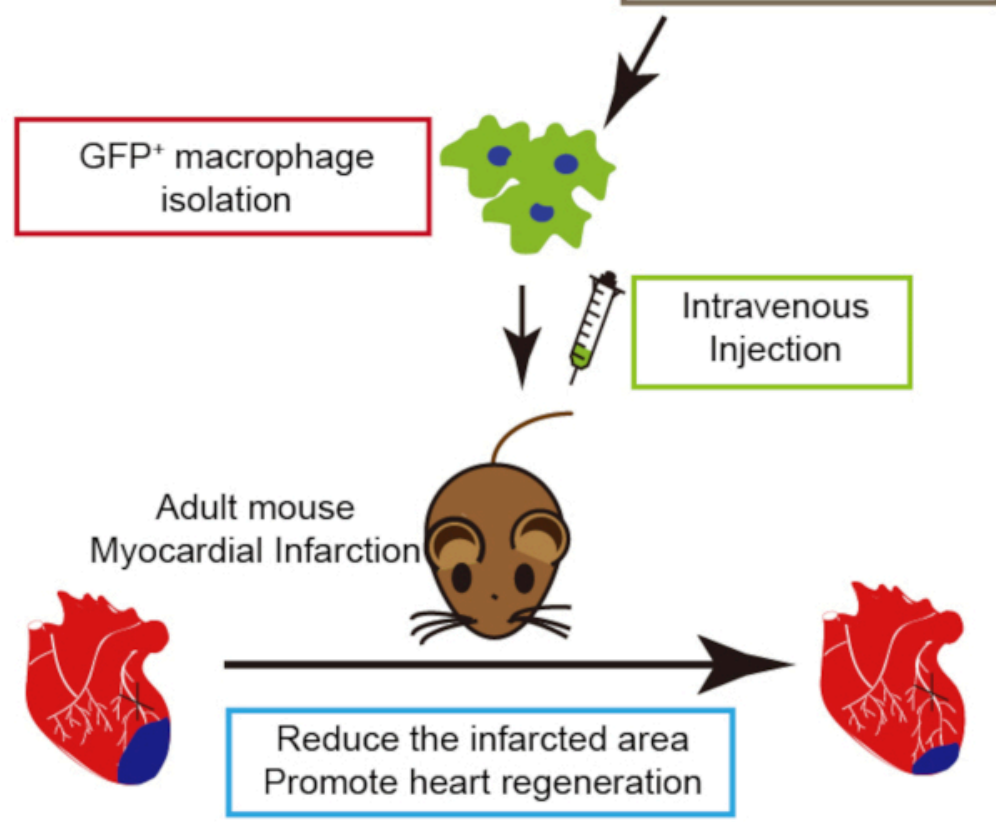

Figure 1: Schematic representation of neonatal cardiac macrophage transplantation. Please click here to view a larger version of this figure. 
A

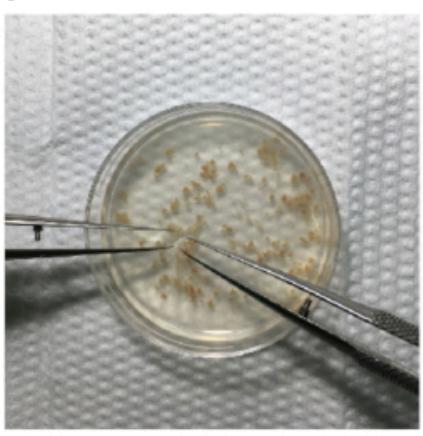

C

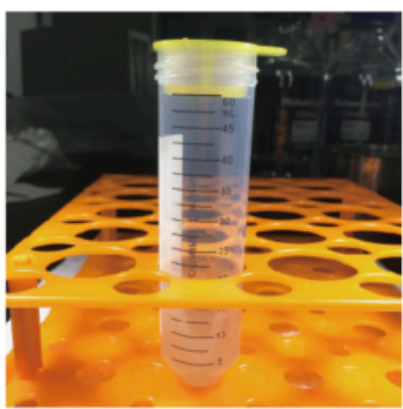

$\mathrm{B}$

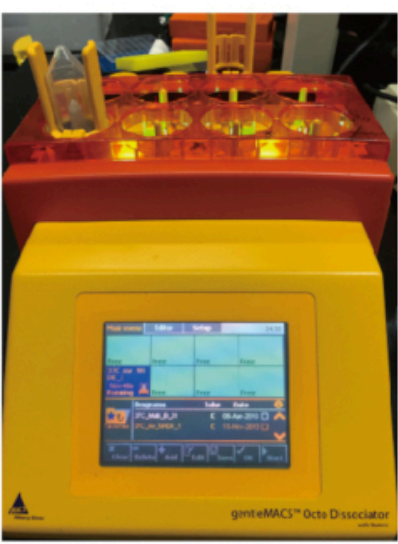

$\mathrm{D}$

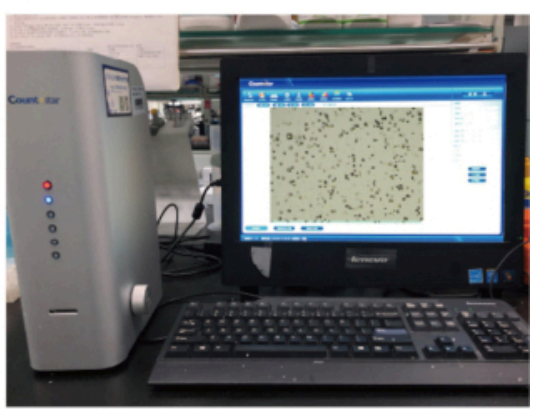

Figure 2: Images of macrophage isolation. A) The hearts are cut into small pieces. B) The mouse hearts are dissociated. C) The suspension is filtered and transferred to a $15 \mathrm{~mL}$ centrifuge tube. D) The number of neonatal cardiac macrophages is calculated. Please click here to view a larger version of this figure. 
A
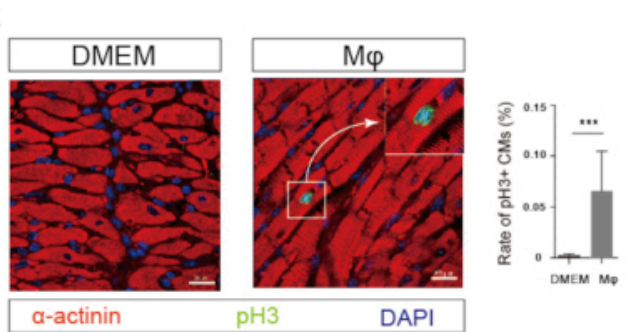

C Analyze 1 month later (1)
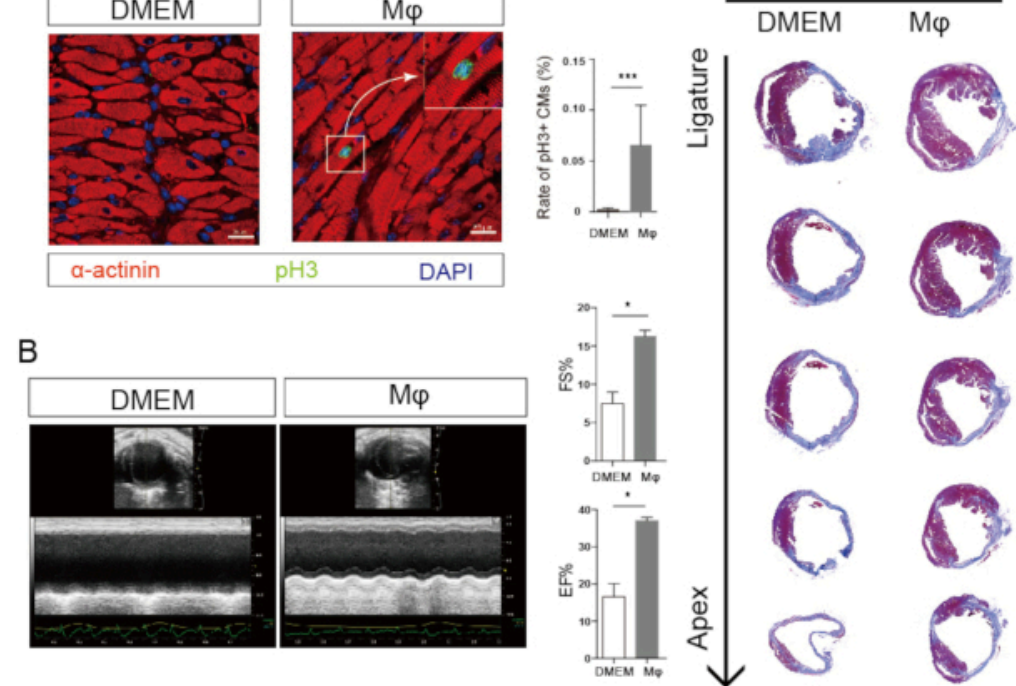

Figure 3: Neonatal cardiac macrophage transplantation promotes adult heart regeneration. A) (Left)

Immunofluorescence images show the proliferative cardiomyocytes (arrow, pH3 green, $\alpha$-actinin red). (Right) Statistical analysis show that cardiomyocyte proliferation increases after macrophage transplantation. B) (Left) Echocardiogram images show the cardiac function in adult mouse 1-month after myocardial infarction. (Right) Statistical analysis show that cardiac function is enhanced after macrophage transplantation. C) Masson's staining shows the infarcted area in adult mouse 1month after myocardial infarction. (Right) Statistical analysis show that infarcted area is reduced after M $\phi$, macrophage transplantation. Please click here to view a larger version of this figure.

\section{Discussion}

Here, we provide an effective approach to prepare, acquire, and transplant neonatal cardiac macrophages to promote adult mouse heart regeneration.

Apical resection is a simple and effective operation to stimulate heart regeneration. We have optimized the details of apical resection to ensure the maximum survival rate of the animals involved in the operation ${ }^{10}$. Anesthesia time should not be longer than 3 minutes, which lead to hypothermiainduced death, or shorter than 2 minutes, which would cause excessive bleeding during the operation. The standard apical resection was supposed to amputate about $1.5 \mathrm{~mm}$ diameter of the apex tissue. However, the purpose of the operation here was to stimulate abundant macrophage infiltration during heart regeneration process. Resection of less than $1.5 \mathrm{~mm}$ diameter was acceptable as it could guarantee a maximum survival rate and effective macrophage recruitment at the same time. Only the skillfulness of the operator influenced the survival rate, and the operated mice could not survive the prolonged operation duration. The operator should complete the whole procedure within 5 minutes.

In our former study, we found that acute inflammation promoted neonatal heart regeneration. Intramyocardial microinjection of immunogenic zymosan A particles into 
neonatal mouse heart could promote cardiomyocyte proliferation $^{6}$. Recently, Molkentin et al. claimed that macrophage infiltration that is stimulated by intracardiac injection of zymosan $A$, cell debris, and freeze/thaw killed cells can promote cardiac repair, confirming that acute inflammation and macrophages are essential in cardiac repair rather than stem cells differentiating into cardiomyocytes ${ }^{11}$. Sadek et al. ${ }^{5}$ reported that macrophages could promote neonatal heart regeneration via angiogenesis. Our recent study revealed that neonatal cardiac macrophage injection could promote adult heart regeneration and increase the ability of adult cardiomyocyte proliferation ${ }^{1,7}$. Neonatal cardiac macrophage transplantation might be a promising strategy to promote adult mouse heart regeneration. Here we present the protocols to help more researchers on the regenerative application development and explore of heart regeneration mechanisms.

\section{Disclosures}

No conflicts of interest.

\section{Acknowledgments}

This work was supported by Chinese Academy of Medical Sciences Innovation Fund for Medical Sciences (CIFMS, 2016-I2M-1-015), the National Key Research and Development Project of China (2019YFA0801500), the National Natural Science Foundation of China (NSFC: 81970243, 81770308), Beijing Natural Science Foundation (7172183, 7182140).

\section{References}

1. Li, Y. et al. gp130 Controls Cardiomyocyte Proliferation and Heart Regeneration. Circulation. 142 (10), 967-982 (2020).
2. Pei, J. et al. Hydrogen Sulfide Promotes Cardiomyocyte Proliferation and Heart Regeneration via ROS Scavenging. Oxidative Medicine and Cellular Longevity. 1412696 (2020).

3. Wang, Y. et al. Mydgf promotes Cardiomyocyte proliferation and Neonatal Heart regeneration. Theranostics. 10 (20), 9100-9112 (2020).

4. Lavine, K.J. et al. Distinct macrophage lineages contribute to disparate patterns of cardiac recovery and remodeling in the neonatal and adult heart. Proceedings of the National Academy of Sciences of the United States of America. 111 (45), 16029-34 (2014).

5. Aurora, A.B. et al. Macrophages are required for neonatal heart regeneration. Journal of Clinical Investigation. 124 (3), 1382-92 (2014).

6. Han, C. et al. Acute inflammation stimulates a regenerative response in the neonatal mouse heart. Cell Research. 25 (10), 1137-51 (2015).

7. $\mathrm{Li}, \mathrm{Y}$. et al. Transplantation of murine neonatal cardiac macrophage improves adult cardiac repair. Cellular \& Molecular Immunology. (2020).

8. Yue, $Z$. et al. PDGFR- $\beta$ Signaling Regulates Cardiomyocyte Proliferation and Myocardial Regeneration. Cell Reports. 28 (4), 966-978.e4 (2019).

9. Wang, J. et al. A long noncoding RNA NR_045363 controls cardiomyocyte proliferation and cardiac repair. Journal of Molecular and Cellular Cardiology. 127, 105-114 (2019).

10. $\mathrm{Li}, \mathrm{Y}$. et al. Achieving stable myocardial regeneration after apical resection in neonatal mice. Journal of Cellular and Molecular Medicine. 24 (11), 6500-6504 (2020). 
11. Vagnozzi, R.J. et al. An acute immune response underlies the benefit of cardiac stem cell therapy. Nature.

577 (7790), 405-409 (2020). 